\title{
Microbiological Profile and Epidemiology of Gram Positive Cocci in Blood Stream Infections in a Tertiary Care Hospital, Kashmir, India
}

\author{
Amrish Kohli, Talat Masoodi, Afreen Rashid*, Sumaira Qayoom, \\ Muzafar Amin and Syed Khurshid \\ Department of Microbiology, SKIMS Medical College, Bemina, \\ Srinagar-190017, J\&K, India \\ *Corresponding author
}

\section{A B S T R A C T}

\section{Keywords}

Bacteremia, Blood culture,

Vancomycin Resistant

Enterococci (VRE),

Methicillin resistant

Staphylococcus

aureus (MRSA)

Article Info

Accepted:

04 April 2019

Available Online:

10 May 2019
Staphylococcus aureus is a virulent pathogen in humans capable of surviving in the otherwise sterile bloodstream and causing a serious and life threatening bacteremia with high morbidity and mortality. Presence in the blood increases its chances of metastasis and the risk of fatality. The choice of antibiotic therapy has conventionally relied to a large extent on the susceptibility of the pathogen to methicillin. In our study we intend to study the cases of bacteremia where Staphylococcus aureus has been isolated and compare it with other gram positive bacteria isolated from blood with reference to their frequency of occurrence and antibiotic sensitivity patterns with main focus on methicillin resistant strains of Staphylococcus aureus.

\section{Introduction}

The infiltration of otherwise sterile bloodstream of compromised hospitalized patients by virulent nosocomial pathogens leads to significant morbidity and mortality. The rapid isolation and antibiotic sensitivity pattern of these organisms causing potentially life threatening infections thus becomes a diagnosis of great importance. ${ }^{[1,2,3]} \mathrm{A}$ consistent presence of microbial pathogen in blood indicates a compromise in the capability of immune system to contain that microbe at its focal site of infection. With the rapid evolution of medical facilities especially among hospitalized patients over the past three decades a change in etiology of bloodstream infections (BSIs), the frequency of isolation of pathogens from blood, their epidemiology and antibiotic sensitivity 
patterns has been observed. More than $50 \%$ of these BSIs are hospital associated. ${ }^{[\mathbf{1}, \mathbf{4}]}$

Trends in the etiology of BSIs have changed over time. The development of potent $\beta$ lactam antimicrobials acting against Staphylococcus aureus made Gram negative bacilli the leading cause of hospital associated infections including BSIs in the 1970s. Soon, early $1980 \mathrm{~s}$ witnessed the reemergence of gram positive cocci in hospital environments that was also reflected in an increase in frequency of their isolation from blood samples of hospitalized patients. Studies conducted at the University of Iowa from 1981 to 1983 associated $52 \%$ and $42 \%$ of the episodes of nosocomial BSIs with gram negative bacilli and gram positive cocci respectively.

However, gram positive cocci accounted for $54 \%$ of such episodes while association of gram negative bacilli with BSIs was reduced to $29 \%$ in the same study from 1990 to 1992 and these trends are still maintained in different parts of the world. ${ }^{[5]}$ Data from 49 hospitals across USA that took part in a project on Surveillance and Control of Pathogens of Epidemiologic Importance (SCOPE) further emphasized the significance of Gram positive cocci as etiologic agents of hospital associated BSIs where $64 \%$ of a total of 10,617 episodes of BSIs occurring over 3 years period were associated with gram positive cocci and only $27 \%$ were caused by gram negative bacilli. ${ }^{[6]}$

Among the gram positive cocci, Staphylococcus aureus and Coagulase negative Staphylococci (CONS) are the most frequent isolates from blood samples. ${ }^{[7]}$ Other causes of BSIs associated with gram positive cocci are Enterococcus spp. and Streptococcus spp. that form important causes of BSIs in particular patient groups like infective endocarditis and neutropenic patients respectively. ${ }^{[8,9]}$ Variations in etiology of Hospital associated BSIs have been observed with the age and location of patient in the hospital. The intensive care patients are likely to harbor CONS while Streptococcus viridians and Staphylococcus aureus are frequently isolated from blood samples of ward patients. The possibility of isolation of Enterococcus species is more from blood samples of patients admitted in surgical ICU as these are more frequently reported pathogens of surgical site infections. ${ }^{[10]}$ Likewise, Enterococcus spp. have been isolated with much greater frequency from blood samples of older age groups of patients that may be associated with the need for more intensive monitoring of geriatric patients with indwelling catheters and invasive devices and age related breaches of skin and mucosa. ${ }^{[11]}$

In the present study, the microbiological profile of gram positive cocci isolated from blood samples was studied and epidemiological factors like age, gender and critical care unit admissions were taken into consideration in relation to the etiological agent isolated. Conventional culture techniques were adopted for isolation of pathogens and culture positive rates were observed. Antibiotic sensitivity pattern of all isolates of gram positive cocci was studied. There was a considerable prevalence of methicillin resistance among Staphylococcus aureus isolates from blood samples in our study.

The present study mainly aims to study the microbiological profile of all gram positive cocci isolated from blood samples of hospitalized patients of bacteremia. To study the isolates of BSIs in relation to demographic parameters like age, gender and location within hospital. And also to study the antibiotic sensitivity pattern and resistance to various drugs. 


\section{Materials and Methods}

\section{Study type and site}

The present study is a retrospective observational study carried out in the department of Microbiology, Sher-i-kashmir institute of medical sciences (SKIMS) Medical college hospital Bemina, Srinagar.

Study period: Three years from March 2016 to March 2019.

Samples: Blood samples from all hospitalized patients of presumptive blood stream infections.

Exclusion criteria: Blood samples of patients hospitalized within 48 hours.

\section{Methodology}

1. Appropriate volume of blood samples were taken from hospitalized patients under strict aseptic precautions and delivered to the microbiology laboratory in brain heart infusion broth.

2. After an initial incubation at $37^{\circ} \mathrm{C}$ for 24 hours, all samples were inoculated on the routine laboratory media like nutrient agar, blood agar, MacConkey agar and chocolate agar following the standard microbiological techniques. ${ }^{[12]}$

3. Any growth obtained after overnight incubation at $37^{\circ} \mathrm{C}$ was put to confirmation by various spot tests like catalase, coagulase and modified oxidase or biochemical tests and hemolytic pattern. ${ }^{[13]}$

4. Antibiotic sensitivity testing of the identified growth of gram positive cocci was performed on Mueller Hinton or Chocolate agar media using the Kirby-Bauer disc diffusion technique according to clinical laboratory standards institute (CLSI) guidelines 2017. ${ }^{[14]}$

The following antibiotics were tested for the isolates of gram positive cocci: Ampicillin $(10 \mu \mathrm{g})$, clindamycin $(2 \mu \mathrm{g})$, erythromycin $(15 \mu \mathrm{g})$, linezolid $(30 \mu \mathrm{g})$, vancomycin $(30 \mu \mathrm{g})$,

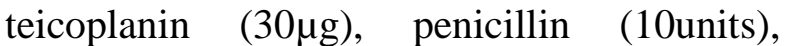
amoxicillin/clavulanic acid $(20 / 10 \mu \mathrm{g})$, amikacin $(30 \mu \mathrm{g}), \quad$ gentamycin $(30 \mu \mathrm{g})$, ciprofloxacin $(5 \mu \mathrm{g})$, co-trimoxazole $(25 \mu \mathrm{g})$, cefoxitin $(30 \mu \mathrm{g})$ and levofloxacin $(5 \mu \mathrm{g})$.

Culture plates with no growth obtained after 48 hours incubation were reincubated till 72 hours and observed for any growth to be processed under steps 2-4. No growth obtained even after 72 hours was labeled as sterile.

\section{Results and Discussion}

A total of 4100 blood samples from hospitalized patients of all age groups were studied in the department of Microbiology Sher-i-Kashmir institute of medical sciences Medical College Bemina between March 2016 and March 2019. Only patients of suspected BSIs hospitalized more than 48 hours were included in the study. Among all the blood samples received, culture showed growth in $632(15.41 \%)$ samples of blood. Rest all samples were reported sterile following 72 hours incubation.

All culture positive cases were studied for individual gram positive cocci and all gram negative bacilli were counted in a single group. Among the culture positive growths, 292 strains of gram negative bacilli $(46.20 \%$ of observable growth) and 337 strains of gram positive cocci (53.32\% of observable growth) were isolated. Three Candida species were also isolated during three years study period and all of them were observed germ tube negative non-albicans Candida. Thus a major 
proportion of all pathogens causing bacteremias in hospitalized patients were gram positive cocci. The culture positivity rates of blood sample are given below in Table 1.

Among all gram positive cocci isolated from the blood samples, coagulase negative Staphylococci formed a major group with 198 isolates out of 337 (58.75\%) followed by 90 isolates of Staphylococcus aureus (26.70\%), 42 isolates of Enterococcus spp. (12.46\%) and 7 isolates of Streptococcus spp. (2.07\%). 6 Streptococcal isolates showed $\alpha$ hemolysis on sheep blood agar (SBA) and on further testing were identified as Streptococcus viridans. One isolate showed $\beta$ hemolysis on SBA medium. A year wise segregation of the etiological agents isolated from blood samples of BSI patients in this institute is given below in Table 2.

All the isolates of gram positive cocci were observed in relation to the epidemiological parameters like the location of the patient within the hospital, the age group and gender of the patient. The results of these observations is depicted below in Table 3. A closer observation of intensive care unit patients for isolation of gram positive cocci revealed the predominance of CONS among them (43.95\%) followed by Enterococcal spp. (35.16\%) and Staphylococcus aureus (18.68\%). However since the overall number of CONS and Staphylococcus aureus isolates was too high from ward patients, their isolation in comparison to wards was only $20 \%$ and $19 \%$ respectively. The prevalence of GPC among ICU patients is given in Figure 1. The pattern of antibiotic resistance during the three year study is given in Table 4 .

\section{Statistics}

Chi-square test was applied for analysis of categorical data. P-value $<0.05$ was taken as significant. Bloodstream infection has become a subject for researchers through its consistently changing spectrum of pathogenic bacteria and their antibiotic sensitivity patterns showing considerable geographic variations. ${ }^{[1,2,3,15,16,17,18,19]}$ A major part of the world has witnessed changing trends in BSIs which includes increasing prevalence of hospital acquired BSIs and increase in the incidence of pathogenic gram positive cocci causing such nosocomial infections. In this study, $54.78 \%$ of the total isolates were found to be Gram positive cocci during the year 2018-19 in comparison to the previous year isolation rate $(50.94 \%)$ suggesting increase in the trend of Gram positive isolation. This trend may be in part due to an increase in the use of third generation cephalosporins, indwelling catheters, intravenous administration of lipid emulsions, injection drug use and enhanced virulence of gram positive cocci in hospitals..$^{[1,3,6,15,20]}$

During our three year study period, an overall culture positive rate of $15.41 \%$ was observed. This finding was well in concordance with the culture positive rates of $16.8 \%, 16.6 \%$ and $16.4 \%$ observed in the studies conducted by Vijaya Devi et al., ${ }^{[21]}$, Qureshi M et al., ${ }^{[22]}$ and Mehta M et al., ${ }^{[23]}$ respectively. However, our results were found discordant with a high culture positivity of $44 \%$ observed in study by Khanal B et al., ${ }^{[2]}$ and a low culture positivity of $7.89 \%$ observed in study by Anbumani $\mathrm{N}$ et al., ${ }^{[25]}$

Among the 337 isolates of gram positive cocci in our study, 198 (58.57\%) were CONS, $90(26.70 \%)$ were Staphylococcus aureus, 42 (12.46\%) were Enterococcus spp. and 7 $(2.07 \%)$ were Streptococcus spp. The high isolation rates of CONS in our study was in concordance with the study conducted by Katyal et al., ${ }^{[26]}$ where $55.5 \%$ of total gram positive cocci were CONS and discordant with the study by Anbumani et al., ${ }^{[25]}$ where 
CONS were only $1.12 \%$ of all gram positive cocci. A higher rate of isolation of Staphylococcus aureus $(21.9 \%)$ was observed compared to CONS (15.6\%) in a study done by Vijay Prakash Singha and Abhishek Mehta $^{[27]}$ that was discordant with the results of our study. CONS are considered common skin commensals and may contaminate the blood samples if proper aseptic precautions are not followed during specimen collection. This might have resulted in an increased isolation of CONS from blood samples in our study. To rule out such contamination and to establish CONS as a true pathogen especially in neonates and geriatric patients, we strongly emphasize the need for clinical correlations and repeat blood cultures in case of isolation of CONS. Yet an important observation in our study was an overall decrease in the isolation of CONS and enterococci from blood samples in 2018-19 compared to the previous year from $40.90 \%$ to $26.26 \%$ and $42.85 \%$ to $28.57 \%$ respectively. This strongly suggests an improvement in the aseptic precautions taken during sample collection and surgical procedures.

The present study also compared various gram positive cocci isolated with respect to the ICU and ward patients, their gender and age groups. A high percent of Enterococcus spp. were isolated from blood samples received from age group more than 50 years (67\% of all enterococcal isolates) and surgical ICU (76\% of all enterococcal isolates). Old age was observed an independent risk factor for acquisition of enterococcal bacteremias in one study ${ }^{[28]}$ Enterococci are well established gut commensals and may spill into the sterile blood during surgical procedures or invasive monitoring of immunocompromised elderly patients with comorbid conditions. CONS, Staphylococcus aureus and Streptococcus spp. were on the contrary isolated more from blood samples received from wards and in pediatric age group patients.
All isolates of gram positive cocci in our study were subject to antibiotic sensitivity testing. An analysis of drug susceptibility of these bacteria over a period of three year indicated prevalence of resistance to some of the important group of antibiotics and an increase in rates of antibiotic resistance over the past three years (Table 4).

Most of the isolates of coagulase negative Staphylococci (CONS) and Staphylococcus aureus were found resistant to amoxicillin/clavulanic acid, cotrimoxazole, azithromycin, erythromycin, levofloxacin, ofloxacin and penicillin. The resistance rate of these two groups of gram positive cocci were comparatively lower for clindamycin $(42 \%$ and $37 \%$ respectively), ciprofloxacin (44\% and $34 \%$ respectively), cefazolin (39\% and $27 \%$ respectively), gentamycin $(31 \%$ and $28 \%$ respectively) and teicoplanin (26\% and $28 \%$ respectively). Most of the isolates of CONS and Staphylococcus aureus were however found sensitive to amikacin (sensitivity rate of $91 \%$ and $81 \%$ respectively).Not a single isolate was found resistant to Linezolid and Vancomycin (sensitivity 100\%).

A considerable number of CONS and Staphylococcus aureus isolates were observed methicillin resistant (66\% CONS and 61\% Staphylococcus aureus). Methicillin resistance has become a worldwide phenomenon especially in hospitalized patients and high rates have been observed in various studies on blood stream infections.

Vibhor Tak et al., ${ }^{[29]}$ in their study observed 59\% of Staphylococcus aureus strains islolated from blood samples as methicillin resistant. In similar studies Parameswaran et al. ${ }^{[7]}$ and Wisplinghoff et al., ${ }^{[30]}$ found $26.7 \%$ and $41 \%$ of their strains resistant to methicillin. In our study we observed a marked increase in the isolation rates of 
MRSA over three years duration from $48 \%$ in 2016-17 to $80 \%$ in $2018-19$. We therefore recommend effective MRSA screening programmes coupled with strict implementation of hospital policies regarding control of infections.

Among the 42 isolates of Enterococcal spp., $13(31 \%)$ were found to be vancomycin resistant. In a study an increase in percentage of nosocomial VRE was observed from $0.3 \%$ to $7.9 \%$ from 1989 through 1993 due to a 34 fold rise of VRE infections in ICU patients with similar trends observed among non-ICU patients as well. ${ }^{[31]}$ However a complete absence of vancomycin resistance was observed in studies by Mohanty et al. ${ }^{[32]}$, Mendiratta et al.., ${ }^{[33]}$ and McBride et al., ${ }^{[34]}$

Table.1 Year wise culture positivity rates of blood samples collected over a three years period

\begin{tabular}{|l|l|l|l|l|}
\hline Laboratory details & Year 2016-2017 & Year 2017-2018 & Year 2018-2019 & Total \\
\hline $\begin{array}{l}\text { Total samples } \\
\text { received }\end{array}$ & 1282 & 1270 & 1548 & 4100 \\
\hline Reported sterile & 1084 & 1066 & 1318 & 3468 \\
\hline Culture growths & $198(\mathrm{CPR}=15.44 \%)$ & $204(\mathrm{CPR}=16.06 \%)$ & $230(\mathrm{CPR}=14.85 \%)$ & $632(\mathrm{CPR}=15.41 \%)$ \\
\hline Gram negative bacilli & $89(44.94 \%)$ & $100(49.01 \%)$ & $103(44.78 \%)$ & $292(46.20 \%)$ \\
\hline Gram positive cocci & $108(54.54 \%)$ & $103(50.49 \%)$ & $126(54.78 \%)$ & $337(53.32 \%)$ \\
\hline Candida spp. & 1 & 1 & 1 & 3 \\
\hline
\end{tabular}

Table.2 Microbiological profile and frequency of isolation of Gram positive cocci isolated from cases of BSIs

\begin{tabular}{|l|l|l|l|l|}
\hline GPC isolated & Year 2016-2017 & Year 2017-2018 & Year 2018-2019 & Total \\
\hline Total isolates & 105 & 118 & 114 & 337 \\
\hline CONS & $65(32.82 \%)$ & $81(40.90 \%)$ & $52(26.26 \%)$ & $198(58.57 \%)$ \\
\hline $\begin{array}{l}\text { Staphylococcus } \\
\text { aureus }\end{array}$ & $33(36.66 \%)$ & $29(32.22 \%)$ & $28(31.11 \%)$ & $90(26.70 \%)$ \\
\hline Enterococcus spp. & $12(28.57 \%)$ & $18(42.85 \%)$ & $12(28.57 \%)$ & $42(12.46 \%)$ \\
\hline Streptococcus spp. & $3(42.85 \%)$ & $2(28.57 \%)$ & $2(28.57 \%)$ & $7(2.07 \%)$ \\
\hline
\end{tabular}

Table.3 Gram positive cocci in relation to the demographic profiles

\begin{tabular}{|l|l|l|l|l|l|l|l|}
\hline \multirow{2}{*}{ GPC isolated } & \multicolumn{2}{|c|}{ Location } & \multicolumn{3}{c|}{ Age group in years } & \multicolumn{2}{c|}{ Gender } \\
\cline { 2 - 8 } & ICU & Ward & $>10$ & $10-50$ & $<50$ & Male & Female \\
\hline CONS & $40(20 \%)$ & $158(80 \%)$ & $137(69 \%)$ & $17(9 \%)$ & $44(22 \%)$ & $104(53 \%)$ & $94(47 \%)$ \\
\hline $\begin{array}{l}\text { Staphylococcus } \\
\text { aureus }\end{array}$ & $17(19 \%)$ & $73(81 \%)$ & $52(58 \%)$ & $15(17 \%)$ & $23(25 \%)$ & $68(76 \%)$ & $22(24 \%)$ \\
\hline Enterococcus spp. & $32(76 \%)$ & $10(24 \%)$ & $9(21 \%)$ & $5(12 \%)$ & $28(67 \%)$ & $14(33 \%)$ & $28(67 \%)$ \\
\hline Streptococcus spp. & $2(29 \%)$ & $5(71 \%)$ & $1(14 \%)$ & $2(29 \%)$ & $4(57 \%)$ & $4(57 \%)$ & $3(43 \%)$ \\
\hline Total & $91(27 \%)$ & $246(73 \%)$ & $199(59 \%)$ & $39(12 \%)$ & $99(29 \%)$ & $190(56 \%)$ & $147(44 \%)$ \\
\hline
\end{tabular}


Table.4 Antibiotic sensitivity pattern of Gram positive cocci from March 2016 to March 2019

\begin{tabular}{|c|c|c|c|c|c|c|c|c|c|c|c|c|c|c|c|c|c|c|}
\hline GPC isolate & Year & $\begin{array}{c}\text { AMC } \\
\text { (R) }\end{array}$ & $\begin{array}{c}\text { AMP } \\
\text { (R) }\end{array}$ & $\begin{array}{l}\text { AK } \\
\text { (R) }\end{array}$ & $\begin{array}{c}\text { AZM } \\
(\mathbf{R})\end{array}$ & $\begin{array}{l}\text { CD } \\
\text { (R) }\end{array}$ & $\begin{array}{c}\text { COT } \\
\text { (R) }\end{array}$ & $\begin{array}{l}\text { CIP } \\
\text { (R) }\end{array}$ & $\begin{array}{l}\mathbf{C Z} \\
\text { (R) }\end{array}$ & $\begin{array}{l}\text { CX } \\
\text { (R) }\end{array}$ & $\begin{array}{c}\mathbf{E} \\
(\mathbf{R})\end{array}$ & $\begin{array}{c}\text { GEN } \\
\text { (R) }\end{array}$ & $\begin{array}{l}\text { LE } \\
\text { (R) }\end{array}$ & $\begin{array}{l}\mathbf{L Z} \\
\text { (R) }\end{array}$ & $\begin{array}{l}\text { OF } \\
\text { (R) }\end{array}$ & $\begin{array}{c}\mathbf{P} \\
\text { (R) }\end{array}$ & $\begin{array}{l}\text { TEI } \\
\text { (R) }\end{array}$ & $\begin{array}{l}\text { VA } \\
\text { (R) }\end{array}$ \\
\hline \multirow[t]{4}{*}{ CONS } & 2016-17 & 82 & 79 & 7 & 88 & 42 & 64 & 46 & 33 & 65 & 68 & 31 & 52 & 0 & 64 & 89 & 19 & 0 \\
\hline & $2017-18$ & 88 & 83 & 5 & 82 & 38 & 63 & 44 & 39 & 68 & 79 & 27 & 45 & 0 & 56 & 91 & 14 & 0 \\
\hline & 2018-19 & 86 & 86 & 14 & 85 & 47 & 53 & 43 & 45 & 66 & 85 & 34 & 61 & 0 & 61 & 90 & 45 & 0 \\
\hline & Total & 85 & 83 & 9 & 85 & 42 & 60 & 44 & 39 & 66 & 77 & 31 & 53 & 0 & 60 & 90 & 26 & 0 \\
\hline \multirow[t]{4}{*}{ S. aureus } & 2016-17 & 81 & nt & 21 & 80 & 33 & 49 & 34 & 18 & 48 & 66 & 22 & 42 & 0 & 62 & 79 & 20 & 0 \\
\hline & 2017-18 & 84 & $\mathrm{nt}$ & 12 & 89 & 17 & 50 & 47 & 12 & 56 & 59 & 12 & 38 & 0 & 56 & 65 & 23 & 0 \\
\hline & 2018-19 & 88 & $\mathrm{nt}$ & 25 & 91 & 60 & 67 & 20 & 52 & 80 & 83 & 50 & 91 & 0 & 100 & 88 & 42 & 0 \\
\hline & Total & 84 & nt & 19 & 87 & 37 & 55 & 34 & 27 & 61 & 69 & 28 & 57 & 0 & 73 & 77 & 28 & 0 \\
\hline \multirow{4}{*}{$\begin{array}{c}\text { Enterococcus } \\
\text { spp. }\end{array}$} & 2016-17 & nt & 68 & 31 & nt & nt & $\mathrm{nt}$ & 57 & nt & nt & 69 & 34 & 49 & 0 & 42 & 68 & 20 & 12 \\
\hline & $2017-18$ & $\mathrm{nt}$ & 55 & 17 & nt & nt & nt & 50 & nt & nt & 58 & 30 & 45 & 0 & 25 & 60 & 12 & 9 \\
\hline & 2018-19 & nt & 75 & 65 & $\mathrm{nt}$ & nt & $\mathrm{nt}$ & 75 & nt & nt & 72 & 50 & 85 & 0 & 64 & 72 & 50 & 17 \\
\hline & Total & nt & 66 & 38 & nt & nt & nt & 61 & nt & nt & 66 & 38 & 60 & 0 & 44 & 67 & 27 & 13 \\
\hline \multirow{4}{*}{$\begin{array}{c}\text { Streptococcus } \\
\text { spp. }\end{array}$} & 2016-17 & 67 & nt & 33 & 67 & 67 & 67 & 67 & 67 & nt & 67 & 33 & 67 & 0 & 33 & 67 & 33 & 0 \\
\hline & $2017-18$ & 50 & nt & 0 & 50 & 100 & 100 & 50 & 100 & nt & 100 & 0 & 50 & 0 & 50 & 50 & 0 & 0 \\
\hline & 2018-19 & 50 & $\mathrm{nt}$ & 50 & 100 & 100 & 50 & 100 & 50 & nt & 100 & 0 & 50 & 0 & 100 & 50 & 0 & 0 \\
\hline & Total & 56 & nt & 28 & 72 & 89 & 72 & 72 & 72 & nt & 89 & 11 & 56 & 0 & 61 & 56 & 11 & 0 \\
\hline
\end{tabular}

Fig.1 Prevalence of gram positive cocci among ICU patients

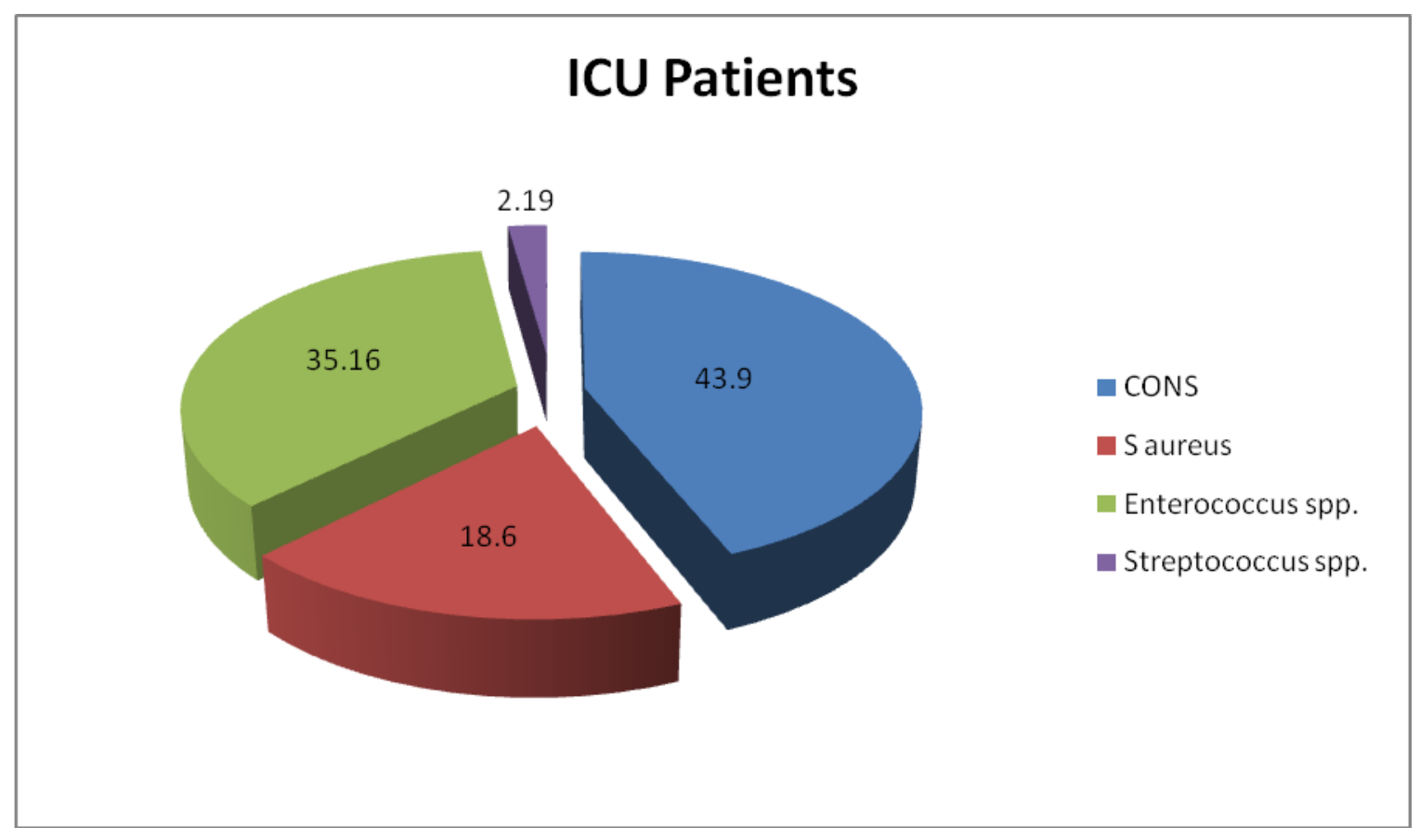

In conclusion, the increased pathogenicity of gram positive cocci combined with their enhanced resistance to the recommended antimicrobials due to intense selection pressure because of excessive use of broad spectrum antibiotics in the hospitals make them a challenge to the clinicians in treatment of life threatening hospital acquired infections. This problem is further aggravated by the increasing number of MRSA and VRE strains isolated from blood samples. A multidisciplinary approach to control the spread of these strains is the need of the hour. This includes strict implementation of 
hospital infection control measures and antimicrobial stewardship programmes.

Conflict of Interest: The authors declare that they have no conflict of interests.

\section{References}

1. Weinstein, MP, Towns, ML, Quartey, SM et al., The clinical significance of positive blood cultures in the 1990s: a prospective comprehensive evaluation of the microbiology, epidemiology and outcome of bacteremia and fungemia in adults. Clin Infect Dis 1997; 24: 584602.

2. Bouza, E, Molina, JP, Munoz, P, Cooperative Group of the European Study Group on Nosocomial Infections (ESGNI). Report of ESGNI-001 and ESGNI-002 studies. Bloodstream infections in Europe. Clin Microbiol Infect 1999; 5(suppl. 2): 1-12.

3. Karchmer, AW. Nosocomial bloodstream infections: organisms, risk factors and implications. Clin Infect Dis 2000;31(suppl 4): 139-43.

4. Bryan CS, Hornung CA, Reynolds KL, Brenner ER. Endemic bacteremia in Columbia, South Carolina, Am J Epidemiol, 1986, vol. 123(pg. 113-27).

5. Pittet D, Wenzel RP. Nosocomial bloodstream infections: secular trends in rates, mortality, and contribution to total hospital deaths, Arch Intern Med, 1995, vol. 155(pg. 1177-84).

6. Edmond MB, Wallace SE, McClish DK, Pfaller MA, Jones RN, Wenzel RP. Nosocomial bloodstream infections in United States hospitals: a 3-year analysis, Clin Infect Dis, 1999, vol. 29 (pg. 239-44).

7. Parameswaran R, Sherchan JB, Varma DM, Mukhopadhyay C, Vidyasagar S. Intravascular catheter-related infections in an Indian tertiary care hospital. J Infect Dev Ctries. 2011; 5: 452-8.

8. Anders Dahl, Trine K. Lauridsen, Magnus Arpi, Lars L. Sorensen, Christian Ostergaard, Peter Sogaard, Niels E. Bruun. Risk Factors of Endocarditis in patients with Enterococcus faecalis Bacteremia: External validation of the NOVA Score. Clin Infect Dis. 2016, vol. 63(6) (pg. 771-775).

9. N Ihendyane, E Sparrelid, B Wretlind, M Remberger, J Andersson, $\mathrm{P}$ Ljungman, O Ringden, B Henriques Normark, U Allen, D E Low, A norrdy Teglund. Viridans streptococcal septicaemia in neutropenic patients: role of proinflammatory cytokines. Bone Marrow Transplantation 33, 79-85 (2004).

10. Richards MJ, Edwards JR, Culver DH, Gaynes RP. Nosocomial infections in combined medical-surgical intensive care units in the United States. Infect Control Hosp Epidemiol. 2000; 21: 5105.

11. Viju Moses, Jayakumar Jerobin, Anupama Nair, Sowmya Sathyendara, Veeraraghavan Balaji, Ige Abraham George, John Victor Peter. Enterococcal Bacteremia is Associated with Prolonged Stay in the Medical Intensive Care Unit. Journal of Global Infectious Diseases. 2012 Jan-Mar;4(1):26-30.

12. Collee J.G., Marr W. Culture of bacteria. In: Collee JG, Fraser AG, Marmion BP, Simmons A (eds). Mackie \& McCartney Practical Medical Microbiology. $14^{\text {th }}$ Ed. London: Churchill Livingstone, 113-129.

13. Collee J.G., Miles R.S., Watt B. Tests for the identification of bacteria. In: Collee JG, Fraser AG, Marmion BP, Simmons A (eds). Mackie \& McCartney Practical Medical 
Microbiology. $14^{\text {th }}$ Ed. London: Churchill Livingstone, 131-149.

14. Clinical and Laboratory Standard Institute. Performance standards for antimicrobial susceptibility testing; $27^{\text {th }}$ edition, CLSI M100-S17. Vol. 37 no.1. Wayne, PA: Clinical and Laboratory Standards Institute; 2017.

15. Bone, RC. Gram-positive organisms and sepsis. Arch Intern Med 1994; 154: 26-34.

16. Yinnon, AM, Schlesinger, Y, Gabbay, D, Rudensky, B. Analysis of 5 years of bacteremias: importance of stratification of microbial susceptibilities by source of patients. J Infect 1997; 35: 17-23.

17. Yucesoy, M, Yuluo, N, Kocagoz, S et al., Antimicrobial resistance of gramnegative isolates from intensive care units in Turkey: a comparison to previous three years. $J$ Chemother 2000 ; 12: 294-8.

18. Phaller, MA, Jones, RN, Doern, GV et al., Survey of bloodstream infections attributable to gram-positive cocci: frequency of occurrence and antimicrobial susceptibility of isolates collected in 1997 in the U.S, Canada, and Latin America from the SENTRY antimicrobial surveillance program. Diagn Microbiol Infect Dis 1999; 33: 283-97.

19. Leibovici, L, Schonheyder, H, Pitlik, SD, Samra, Z, Moller, JK. Bacteremia caused by hospital-type microorganisms during hospital stay. J Hosp Infect 2000; 44: 31-6.

20. Warren, DK, Zack, JE, Elward, AM, Cox, MJ, Fraser, VJ. Nosocomial primary bloodstream infections in intensive care unit patients in a nonteaching community medical center: a 21-month prospective study. Clin Infect Dis 2001; 33: 1329-35.

21. Vijaya Devi A, Sahoo B, Damrolien S, Praveen SH, Lungran P, Ksh Mamta
Devi. A Study on the Bacterial Profile of Bloodstream Infections in Rims Hospital. (IOSR-JDMS). 2015; 14(1): 18-23.

22. Qureshi M, Aziz F. Prevalence of microbial isolates in blood culture and their antimicrobial susceptibility profile. Biomedica. 2011; 27: 136-39.

23. Mehta M, Pyria D, Varsha G. Antimicrobial susceptibility pattern of blood isolates from a teaching Hospital in north India. Japan J Infec Dis. 2005; 58:174-176.

24. Khanal B, Harish BN, Sethuraman KR, Srinivasan S. Infective endocarditis: Report of prospective study in an Indian Hospital. Trop Doct 2002; 32:83-85.

25. Anbumani N, Kalyani J, Mallika M. Original research distribution and antimicrobial susceptibility of bacteria isolated from blood cultures of hospitalized patients in a tertiary care hospital. Indian Journal for the practicing doctor 2008;5(2).

26. Katyal A, Singh D, Sharma M, Chaudhary U. Bacteriological profile and antibiogram of aerobic blood culture isolates from intensive care units in a Teaching Tertiary Care Hospital. $J$ Health Science Res 2018 9(1): 6-10.

27. Vijay Prakash Singha, Abhishek Mehtab. Bacteriological profile of bloodstream infections at a Rural tertiary care teaching hospital of Western Uttar Pradesh. Indian Journal of Basic and Applied Medical Research; June 2017: Vol.-6, Issue- 3, P. 393-401.

28. Chatterjee I, Dulhunty JM, Iredell J, Gallagher JE, Sud A, Woods M, et al., Predictors and outcome associated with an Enterococcus positive isolate during intensive care unit admission. Anaesth Intensive Care. 2009; 37: 976-82.

29. Vibhor Tak, Purva Mathur, Sanjeev Lalwani, Mahesh Chandra Misra. Staphylococcal Blood Stream 
Infections: Epidemiology, Resistance Pattern and Outcome at a Level 1 Indian Trauma Care Center. J Lab Physicians. 2013 Jan-Jun; 5(1): 46-50.

30. Vibhor Tak, Purva Mathur, Sanjeev Lalwani, Mahesh Chandra Misra. Staphylococcal Blood Stream Infections: Epidemiology, Resistance Pattern and Outcome at a Level 1 Indian Trauma Care Center. J Lab Physicians. 2013 Jan-Jun; 5(1): 46-50.

31. Yesim Cetinkaya, Pamela Falk, C. Glen Mayhall. Vancomycin-Resistant Enterococci. Clinical Microbiology Reviews. DOI: 10.1128/CMR.13.4.686.

32. Mohanty S, Kapil A, Das BK. Enterococcal bacteremia in a tertiary care hospital of North India. J Indian Med Assoc. 2005;103:31-7.

33. Mendiratta DK, Kaur H, Deotale V, Thamke DC, Narang R, Narang P. Status of high level aminoglycoside resistant Enterococcus faecium and Enterococcus faecalis in a rural hospital of central India. Indian $\mathrm{J}$ Med Micriobiol. 2008;26:369-71.

34. McBride SJ, Upton A, Roberts SA. Clinical characteristics and outcomes of patients with vancomycin-susceptible Enterococcus faecalis and Enterococcus faecium bacteremia-a five year retrospective review. Eur J Clin Microbiol Infect Dis. 2010;29:107-14.

\section{How to cite this article:}

Amrish Kohli, Talat Masoodi, Afreen Rashid, Sumaira Qayoom, Muzafar Amin and Syed Khurshid. 2019. Microbiological Profile and Epidemiology of Gram Positive Cocci in Blood Stream Infections in a Tertiary Care Hospital, Kashmir, India. Int.J.Curr.Microbiol.App.Sci. 8(05): 58-67. doi: https://doi.org/10.20546/ijcmas.2019.805.008 